\title{
The boundedness of Fourier transform on the Herz type amalgams and Besov spaces
}

Jiang Zhou and Yonghui Cao*

${ }^{*}$ Correspondence:

m13009688600@163.com

College of Mathematics and System

Science, Xinjiang University, Urumqi,

830046, P.R. China

\begin{abstract}
In this paper, using the Young inequality as regards the Herz space, the authors obtain the boundedness of the Fourier transform on the Herz type amalgams and Besov spaces. As corollaries, the authors get the estimates of the Fourier transform on the weighted amalgams and Besov spaces.
\end{abstract}

MSC: 42B10; 42B35

Keywords: Fourier transform; Herz-Besov space; Herz-amalgam space; weighted

\section{Introduction}

One of the central problems in classic harmonic analysis is the study of the boundedness of Fourier transform on a given function space. A basic result is the Hausdorff-Young inequality and its various extensions and generalizations. Here and below we define the Fourier transform of $f \in L^{1} \cap L^{p}$ to be

$$
\mathcal{F} f(x)=\int_{\mathbf{R}^{n}} f(y) e^{-i y x} d y .
$$

When $1 \leq p \leq 2, \mathcal{F}$ is bounded from $L^{p}$ to $L^{p^{\prime}}$. If the spaces are weighted Lebesgue spaces or Herz spaces, the following results are proved (see [1-4]).

Theorem A If $1<p \leq 2$ and $0 \leq \alpha<\frac{1}{p^{\prime}}$, then

$$
\left(\int_{\mathbf{R}^{n}}|\mathcal{F} f|^{p^{\prime}}|x|^{-\alpha n p^{\prime}} d x\right)^{\frac{1}{p^{\prime}}} \leq c\left(\int_{\mathbf{R}^{n}}|f|^{p}|x|^{\alpha n p} d x\right)^{\frac{1}{p}} .
$$

Theorem B If $1<p \leq 2$ and $0 \leq \alpha<\frac{1}{p^{\prime}}$, then

$$
\|\mathcal{F} f\|_{\dot{K}_{2}^{-\alpha, p^{\prime}}} \leq c\|f\|_{\dot{K}_{p}^{\alpha, p}}
$$

Here $\dot{K}_{q}^{\alpha, p}$ denotes a Beurling-Herz space.

In [5], the authors give a result as regards the Young theorem for amalgams and Besov spaces.

Theorem C (1) Let $1 \leq p \leq 2,0<q \leq \infty$ and $s \in \mathbf{R}$. Then

$$
\mathcal{F}:\left(L^{p}, l^{q}\left(\langle z\rangle^{s}\right)\right) \rightarrow B_{p^{\prime}, q}^{s-n\left(\frac{1}{p}-\frac{1}{q}\right)_{+}} .
$$

O2014 Zhou and Cao; licensee Springer. This is an Open Access article distributed under the terms of the Creative Commons Attribution License (http://creativecommons.org/licenses/by/2.0), which permits unrestricted use, distribution, and reproduction in any medium, provided the original work is properly cited. 
(2) Let $1 \leq p \leq 2,0<q \leq \infty$ and $s \in \mathbf{R}$. Then

$$
\mathcal{F}: B_{p, q}^{s} \rightarrow\left(L^{p^{\prime}}, l^{q}\left(\langle z\rangle^{s-n\left(\frac{1}{q}-\frac{1}{p^{\prime}}\right)_{+}}\right)\right)
$$

Inspired by Theorem B and Theorem C, in this paper, we discuss the boundedness as regards the Fourier transform on the Herz type amalgams and the Besov spaces. In Section 2, we give the definition of function spaces. The main theorems and their proofs are contained in Section 3.

\section{Function spaces}

In this section, we give the definition of function spaces that we work on. Let $\mathcal{S}=\mathcal{S}\left(\mathbf{R}^{n}\right)$ and $\mathcal{S}^{\prime}=\mathcal{S}^{\prime}\left(\mathbf{R}^{n}\right)$ be a Schwartz space and its dual space.

Definition 2.1 (Weighted Lebesgue spaces) Let $\omega(x)$ be a nonnegative function, $L^{p}(\omega)=$ $\left\{f(x) \mid\|f\|_{L^{p}(w)}<\infty\right\}$, where

$$
\|f\|_{L^{p}(w)}=\left(\int_{\mathbf{R}^{n}}|f|^{p} \omega(x) d x\right)^{\frac{1}{p}}
$$

Now we give the definitions as regards Herz spaces.

Definition 2.2 Suppose $-\infty<\alpha<\infty, 0<p \leq \infty, 0<q \leq \infty$. The Herz space $\dot{K}_{q}^{\alpha, p}$ is defined by

$$
\dot{K}_{q}^{\alpha, p}=\left\{f \in L_{\mathrm{loc}}^{q}\left(\mathbf{R}^{n} \backslash\{0\}\right):\|f\|_{\left.K_{q}^{\alpha, p}<\infty\right\}}\right.
$$

where $\mathcal{X}_{B_{k}}(x)=\mathcal{X}_{C_{k}-C_{k-1}}(x), C_{k}=\left\{x \in \mathbf{R}^{n}:|x| \leq 2^{k}\right\}$,

$$
\|f\|_{\dot{K}_{q}^{\alpha, p}}=\left\{\sum_{k=-\infty}^{\infty} 2^{k \alpha q}\left\|f \chi_{B_{k}}\right\|_{L^{p}}^{q}\right\}^{1 / q} .
$$

The usual modification should be made when $p=\infty$ or $q=\infty$ (see [6]).

In order to define the Herz type Besov space, we need the following Littlewood-Paley function (see $[5,7])$.

Definition 2.3 Let $\phi_{0}, \phi_{1} \in \mathcal{S}$ be even functions satisfying the following condition:

$$
\mathcal{X}_{[-2,2]^{n}} \leq \phi_{0} \leq \mathcal{X}_{[-4,4]^{n}}, \quad \mathcal{X}_{[-4,4]^{n}-[-2,2]^{n}} \leq \phi_{1} \leq \mathcal{X}_{[-8,8]^{n}-[-1,1]^{n}}
$$

We set $\phi_{j}(x)=\phi_{1}\left(2^{-j+1} x\right)$ for $j \geq 2$.

For $f \in \mathcal{S}^{\prime}$, we denote $\phi_{j}(D) f=\mathcal{F}^{-1}\left(\phi_{j} \mathcal{F} f\right)$. Now let us introduce the Herz type Besov spaces (see [8]).

Definition 2.4 Let $-\infty<\alpha<\infty, 0<p<\infty, 1 \leq q<\infty$, and $-\infty<s<\infty, 0<r \leq \infty$; then we define

$$
\dot{K}_{q}^{\alpha, p} B_{r}^{s}=\left\{f \in S^{\prime} \mid\|f\|_{\dot{K}_{q}^{\alpha, p} B_{r}^{s}}=\left\{\sum_{i=0}^{\infty} 2^{i s r}\left\|\phi_{j}(D) f\right\|_{\dot{K}_{q}^{\alpha, p}}^{r}\right\}^{1 / r}<\infty\right\} .
$$


Obviously, the definition of the Herz-Besov space is independent of the choice of $\phi_{0}, \phi_{1}$ and $\dot{K}_{p}^{0, p} B_{r}^{s}=B_{p, r}^{s}$, standard Besov spaces. More information as regards the Besov space and Herz type Besov spaces can be found in [7, 9-12].

Definition 2.5 (Herz type amalgam space) Let $-\infty<\alpha<\infty, 0<p<\infty, 1 \leq q<\infty, s \in \mathbf{R}$, and $0<r \leq \infty$. Set $Q_{z}=z+[0,1]^{n}$, the translation of the unit cube. For a Lebesgue locally integrable function $f$ we define

$$
\|f\|_{\left(\dot{K}_{q}^{\alpha, p}, r^{r}\left(\langle z)^{s}\right)\right)}=\left\|\left\{\langle z\rangle^{s}\left\|f \mathcal{X}_{Q_{z}}\right\|_{\dot{K}_{q}^{\alpha, p}}\right\}\right\|_{l^{r}}
$$

where $\langle z\rangle=\sqrt{|z|^{2}+1} .\left(\dot{K}_{q}^{\alpha, p}, l^{r}\left(\langle z\rangle^{s}\right)\right)$ is the set of all locally integrable functions $f$ for which the quasi-norm $\|f\|_{\left(K_{q}^{\alpha, p}, r^{r}\left(\langle z)^{s}\right)\right)}<\infty$.

If $\alpha=0$ and $p=q$ in Definition 2.5, then the Herz type amalgam space is also an amalgam space $\left(L^{p}, l^{r}\left(\langle z\rangle^{s}\right)\right)($ see $[5,13,14])$.

\section{Main theorems and proofs}

In this section we formulate our main theorems and proofs.

Theorem 3.1 Let $1<p \leq 2,0<q \leq \infty, 0 \leq \alpha<\frac{1}{p^{\prime}}$ and $s \in \mathbf{R}$. Then the Fourier transform is bounded from the Herz type amalgam space $\left(\dot{K}_{p}^{\alpha, p}, l^{q}\left(\langle z\rangle^{s}\right)\right)$ to the Herz type Besov space $\dot{K}_{2}^{-\alpha, p^{\prime}} B_{q}^{s-n\left(\frac{1}{p}-\frac{1}{q}\right)_{+}}$. Here and below, for $a \in \mathbf{R}$ we write $a_{+}=\max \{a, 0\}$.

Proof of Theorem 3.1 If we note that the lift operator $(I-\triangle)^{\frac{t}{2}}$ is bounded from $\dot{K}_{u}^{\alpha, p} B_{q}^{s}$ to $\dot{K}_{u}^{\alpha, p} B_{q}^{s-t}$ (see [8]) and the following multiplication operator is an isomorphism:

$$
f \in\left(\dot{K}_{q}^{\alpha, p}, l^{r}\left(\langle z\rangle^{s}\right)\right) \mapsto\langle\cdot\rangle^{t} \cdot f \in\left(\dot{K}_{q}^{\alpha, p}, l^{r}\left(\langle z\rangle^{s-t}\right)\right),
$$

we can assume $s=0$.

Let $A_{j}=\operatorname{supp}\left(\phi_{j}\right), j \in \mathbf{N}_{0}$, where $\phi_{j}$ is the Littlewood-Paley function. Using the Young inequality $\|\mathcal{F} f\|_{\dot{K}_{2}^{-\alpha, p^{\prime}}} \leq c\|f\|_{\dot{K}_{p}^{\alpha, p}, \text { we obtain }}$

$$
\begin{aligned}
\left\|2^{-j n\left(\frac{1}{p}-\frac{1}{q}\right)_{+}} \phi_{j}(D) \mathcal{F} f\right\|_{\dot{K}_{2}^{-\alpha, p^{\prime}}} & \leq\left\|2^{-j n\left(\frac{1}{p}-\frac{1}{q}\right)_{+}} \phi_{j} \mathcal{F} \mathcal{F} f\right\|_{\dot{K}_{p}^{\alpha, p}} \\
& \leq c\left\|2^{-j n\left(\frac{1}{p}-\frac{1}{q}\right)_{+}} \phi_{j} f(-x)\right\|_{\dot{K}_{p}^{\alpha, p}} \\
& \leq c\left\|2^{-j n\left(\frac{1}{p}-\frac{1}{q}\right)_{+}} \mathcal{X}_{A_{j}} f\right\|_{\dot{K}_{p}^{\alpha, p} .}
\end{aligned}
$$

But

$$
\begin{aligned}
\left\|\mathcal{X}_{A_{j}} f(x)\right\|_{\dot{K}_{p}^{\alpha, p}}^{q} & \leq\left(\sum_{k=-\infty}^{\infty} 2^{k \alpha p}\left\|\mathcal{X}_{B_{k}} \mathcal{X}_{A_{j}} f\right\|_{p}^{p}\right)^{\frac{q}{p}} \\
& \leq c\left(\sum_{k=-\infty}^{\infty} 2^{k \alpha p} \sum_{z \in \mathbf{Z}^{n}}\left\|\mathcal{X}_{B_{k}} \mathcal{X}_{\mathrm{Q}_{z}} \mathcal{X}_{A_{j}} f\right\|_{p}^{p}\right)^{\frac{q}{p}}
\end{aligned}
$$




$$
\begin{aligned}
& \leq c\left(\sum_{z \in \mathbf{Z}^{n}}\left(\sum_{k=-\infty}^{\infty} 2^{k \alpha p}\left\|\mathcal{X}_{B_{k}} \mathcal{X}_{Q_{z}} \mathcal{X}_{A_{j}} f\right\|_{p}^{p}\right)\right)^{\frac{q}{p}} \\
& \leq c\left(\sum_{|z| \backsim 2^{j n}}\left\|\mathcal{X}_{Q_{z}} \mathcal{X}_{A_{j}} f\right\|_{\dot{K}_{p}^{\alpha, p}}^{p}\right)^{\frac{q}{p}} \\
& \leq c 2^{j n\left(\frac{q}{p}-1\right)_{+}} \sum_{|z| \backsim 2^{j n}}\left\|\mathcal{X}_{Q_{z}} \mathcal{X}_{A_{j}} f\right\|_{\dot{K}_{p}^{\alpha, p}}^{q}
\end{aligned}
$$

where we use the inequality $\left(\sum_{j=1}^{L}\left|a_{j}\right|\right)^{\frac{q}{p}} \leq L^{\left(\frac{q}{p}-1\right)_{+}} \sum_{j=1}^{L}\left|a_{j}\right|^{\frac{q}{p}}$ (see [5]).

If we put these estimates together, we have

$$
\begin{aligned}
\left\|\left\{\left\|2^{-j n\left(\frac{1}{p}-\frac{1}{q}\right)_{+}} \phi_{j}(D) \mathcal{F} f\right\|_{\dot{K}_{2}^{-\alpha, p^{\prime}}}\right\}\right\|_{l q} & \leq c\left\|\left\{\left(\sum_{z}\left\|\mathcal{X}_{A_{j}} \mathcal{X}_{Q_{z}} f\right\|_{\dot{K}_{p}^{\alpha, p}}^{q}\right)^{\frac{1}{q}}\right\}\right\|_{l q} \\
& \leq c\left(\sum_{z} \sum_{j}\left\|\mathcal{X}_{A_{j}} \mathcal{X}_{Q_{z}} f\right\|_{\dot{K}_{p}^{\alpha, p}}^{q}\right)^{\frac{1}{q}} .
\end{aligned}
$$

Given $z \in \mathbf{Z}^{n}$, from the definition of the $\phi_{j}$, there are at most three $j$ such that $A_{j} \cap Q_{z} \neq \emptyset$. So we have

$$
\begin{aligned}
\left\|\left\{\left\|2^{-j n\left(\frac{1}{p}-\frac{1}{q}\right)_{+}} \phi_{j}(D) \mathcal{F} f\right\|_{\dot{K}_{2}^{-\alpha, p^{\prime}}}\right\}\right\|_{l^{q}} & \leq c\left(\sum_{z} \sum_{j}\left\|\mathcal{X}_{A_{j}} \mathcal{X}_{Q_{z}} f\right\|_{\dot{K}_{p}^{\alpha, p}}^{q}\right)^{\frac{1}{q}} \\
& \leq c\left(\sum_{z}\left\|\mathcal{X}_{Q_{z}} f\right\|_{\dot{K}_{p}^{\alpha, p}}^{q}\right)^{\frac{1}{q}} \\
& \leq c\|f\|_{\left(\dot{K}_{p}^{\alpha, p}, l q\left(\langle z)^{s}\right)\right)^{*}}
\end{aligned}
$$

This is the desired result.

Theorem 3.2 Let $1<p \leq 2,0<q \leq \infty, 0 \leq \alpha<\frac{1}{p^{\prime}}$ and $s \in \mathbf{R}$. Then the Fourier transform is bounded from the Herz type Besov space $\dot{K}_{p}^{\alpha, p} B_{q}^{s}$ to the Herz type amalgams space $\left(\dot{K}_{2}^{-\alpha, p^{\prime}}, l^{q}\left(\langle z\rangle^{s-n\left(\frac{1}{q}-\frac{1}{p^{\prime}}\right)_{+}}\right)\right)$.

Proof of Theorem 3.2 As before, we assume $s=0$. Let $|z|_{\infty}=\max \left\{\left|z_{1}\right|, \ldots,\left|z_{n}\right|\right\}$. By the definition of the Herz type amalgam space, we have

$$
\|\mathcal{F} f\|_{\left(\dot{K}_{2}^{-\alpha, p^{\prime}}, l q(\langle z\rangle\right.}^{\left.\left.q-n\left(\frac{1}{q}-\frac{1}{p^{\prime}}\right)_{+}\right)\right)} \sim\left\|\left\{2^{-j n q\left(\frac{1}{q}-\frac{1}{p^{\prime}}\right)_{+}} \sum_{|z|_{\infty} \backsim 2^{j n}}\|\mathcal{F} f\|_{\dot{K}_{2}^{-\alpha, p^{\prime}}}^{q}\right\}\right\|_{l^{1}} .
$$

When $|z|_{\infty} \backsim 2^{j}$, from the definition of $B_{k}$, there are at most three $k(k=j-1, j, j+1)$ such that $Q_{z} \cap B_{k} \neq \emptyset$. If $\left|z_{1}\right|_{\infty}, \ldots,\left|z_{i}\right|_{\infty} \backsim 2^{j}$ and $Q_{z_{1}} \cap \cdots \cap Q_{z_{i}}=\emptyset$, we have

$$
\begin{aligned}
& \left\|\mathcal{X}_{Q_{z_{1}}} \mathcal{F} f\right\|_{\dot{K}_{2}^{-\alpha, p^{\prime}}}^{p^{\prime}}+\cdots+\left\|\mathcal{X}_{Q_{z_{i}}} \mathcal{F} f\right\|_{\dot{K}_{2}^{-\alpha, p^{\prime}}}^{p^{\prime}} \\
& \quad \leq c \sum_{k=j-1}^{j+1} 2^{-k \alpha p^{\prime}}\left\|\mathcal{X}_{Q_{z_{1}}} \mathcal{X}_{B_{k}} \mathcal{F} f\right\|_{p^{\prime}}^{p^{\prime}}+\cdots+c \sum_{k=j-1}^{j+1} 2^{-k \alpha p^{\prime}}\left\|\mathcal{X}_{Q_{z_{i}}} \mathcal{X}_{B_{k}} \mathcal{F} f\right\|_{p^{\prime}}^{p^{\prime}}
\end{aligned}
$$




$$
\begin{aligned}
& =c \sum_{k=j-1}^{j+1} 2^{-k \alpha p^{\prime}}\left\|\mathcal{X}_{Q_{z_{1}} \cup \cdots \cup Q_{z_{i}}} \mathcal{X}_{B_{k}} \mathcal{F} f\right\|_{p^{\prime}}^{p^{\prime}} \\
& =c\left\|\mathcal{X}_{Q_{z_{1}} \cup \ldots \cup Q_{z_{i}}} \mathcal{F} f\right\|_{\dot{K}_{2}^{-\alpha, p^{\prime}}}^{p^{\prime}}
\end{aligned}
$$

Using the above inequality and $\sum_{i=1}^{L}\left|a_{i}\right|^{\frac{q}{p^{\prime}}} \leq L^{\left(1-\frac{q}{p^{\prime}}\right)+}\left(\sum_{i=1}^{L}\left|a_{i}\right|\right)^{\frac{q}{p^{\prime}}}$ (see [5]) we have

$$
\begin{aligned}
2^{-j n q\left(\frac{1}{q}-\frac{1}{p^{\prime}}\right)_{+}} \sum_{|z|_{\infty} \backsim 2^{j n}}\|\mathcal{F} f\|_{\dot{K}_{2}^{-\alpha, p^{\prime}}}^{q} & \leq 2^{-j n q\left(\frac{1}{q}-\frac{1}{p^{\prime}}\right)+} \sum_{|z|_{\infty} \sim 2^{j n}}\|\mathcal{F} f\|_{\dot{K}_{2}^{-\alpha, p^{\prime}}}^{p^{\prime}} \\
& \leq\left(\sum_{|z|_{\infty} \sim 2^{j n}}\|\mathcal{F} f\|_{\dot{K}_{2}^{-\alpha, p^{\prime}}}^{p^{\prime}}\right)^{\frac{q}{p^{\prime}}} \backsim\left\|\mathcal{X}_{B_{j}} \mathcal{F} f\right\|_{\dot{K}_{2}^{-\alpha, p^{\prime}}}^{q} .
\end{aligned}
$$

So

$$
\begin{aligned}
\left.\left.\|\mathcal{F} f\|_{\left(\dot{K}_{2}^{-\alpha, p^{\prime}}, l q((z)\right.}-n\left(\frac{1}{q}-\frac{1}{p^{\prime}}\right)_{+}\right)\right) & \leq\left\|\left\{\left\|\mathcal{X}_{B_{j}} \mathcal{F} f\right\|_{\dot{K}_{2}^{-\alpha, p^{\prime}}}\right\}\right\|_{l^{q}} \\
& \leq c\left\|\left\{\left\|\phi_{j} \mathcal{F} f\right\|_{\dot{K}_{2}^{-\alpha, p^{\prime}}}\right\}\right\|_{l^{q}} \\
& \leq c\left\|\left\{\left\|\phi_{j}(D) f\right\|_{\dot{K}_{p}^{\alpha, p}}\right\}\right\|_{l^{q}} \\
& \leq c\|f\|_{\dot{K}_{p}^{\alpha, p} B_{q}^{0} .}
\end{aligned}
$$

This proves Theorem 3.2.

We note that $\dot{K}_{p}^{\alpha, p}=L^{p}\left(|x|^{\alpha}\right)$ (see [2]) and $\dot{K}_{2}^{\alpha, p} \subset \dot{K}_{q}^{\alpha, p}, q \geq 2$. Then we have the following.

Corollary 3.3 Let $1<p \leq 2,0<q \leq \infty, 0 \leq \alpha<\frac{1}{p^{\prime}}$, and $s \in \mathbf{R}$. Then the Fourier transform is bounded from the weighted amalgam space $\left(L^{p}\left(|x|^{\alpha}\right), l^{q}\left(\langle z\rangle^{s}\right)\right)$ to the weighted Besov space $B_{p^{\prime}, q}^{s-n\left(\frac{1}{p}-\frac{1}{q}\right)_{+}}\left(|x|^{-\alpha}\right)$.

Corollary 3.4 Let $1<p \leq 2,0<q \leq \infty, 0 \leq \alpha<\frac{1}{p^{\prime}}$, and $s \in \mathbf{R}$. Then the Fourier transform is bounded from the weighted Besov space $B_{p, q}^{s}\left(|x|^{\alpha}\right)$ to the weighted amalgam space $\left(L^{p^{\prime}}\left(|x|^{-\alpha}\right), l^{q}\left(\langle z\rangle^{s-n\left(\frac{1}{q}-\frac{1}{p^{\prime}}\right)_{+}}\right)\right)$.

If $q=p$ and $s=0$ in Theorem 3.1, we have the following.

Corollary 3.5 Let $1<p \leq 2$ and $0 \leq \alpha<\frac{1}{p^{\prime}}$. Then the Fourier transform is bounded from the Herz space $\dot{K}_{p}^{\alpha, p}$ to the Herz type Besov space $\dot{K}_{2}^{-\alpha, p^{\prime}} B_{p}^{0}$.

Next we deduce some necessary condition as regards the boundedness of the Fourier transform.

Proposition 3.6 Let $1 \leq p_{1} \leq \infty, 0<p_{2}, q_{1}, q_{2}, u_{1}, u_{2} \leq \infty, \alpha_{1} \geq 0$, and $\alpha_{2}, s_{1}, s_{2} \in \mathbf{R}$. If

$$
\|\mathcal{F} f\|_{\dot{K}_{u_{2}}^{\alpha_{2}, p_{2}} B_{q_{2}}^{s_{2}}} \leq c\|f\|_{\left(\dot { K } _ { u _ { 1 } } ^ { \alpha _ { 1 } , p _ { 1 } , q _ { 1 } } \left(\langle z)^{\left.\left.s_{1}\right)\right)}\right.\right.},
$$

then $p_{1} \geq p_{2}^{\prime}$. 
Proof of Proposition 3.6 Let $\theta \in \mathbf{S}$ be an even function with $\mathcal{X}_{B\left(0, \frac{1}{4}\right)} \leq \theta \leq \mathcal{X}_{B\left(0, \frac{1}{2}\right)}$. We take $f(x)=|x|^{-\alpha_{1}} \theta, g(x)=|x|^{-\alpha_{1}}(1-\theta)$. So $\mathcal{F}(f+g)(x)=c|x|^{\alpha_{1}-n}$. Since $|x|^{2 v} \mathcal{F} g(x)=$ $\mathcal{F}\left((-\Delta)^{v} g\right)(x)<c(v \gg 1)$, it follows that $|\mathcal{F} g(x)| \leq c|x|^{-2 v}$. From this we have $|\mathcal{F} f(x)| \backsim$ $|x|^{\alpha_{1}-n}(x \rightarrow \infty)$.

Since supp $f \subset B\left(0, \frac{1}{2}\right)$, we have $\|f\|_{\left(\dot{K}_{u_{1}}^{\alpha_{1}, p_{1}, q_{1}}\right.}\left[(z)^{\left.\left.s_{1}\right)\right)}\right)\|f\|_{\dot{K}_{u_{1}}^{\alpha_{1}, p_{1}}}$. But $\left\|f \mathcal{X}_{B_{k}}\right\|_{p_{1}} \backsim 2^{\frac{k}{p_{1}}-\alpha_{1} k}$. So

$$
\begin{aligned}
\|f\|_{\dot{K}_{u_{1}}^{\alpha_{1}, p_{1}}} & \backsim\left(\sum_{k=-\infty}^{1} 2^{k \alpha_{1} u_{1}}\left\|f \mathcal{X}_{B_{k}}\right\|_{p_{1}}^{u_{1}}\right)^{\frac{1}{u_{1}}} \\
& \backsim \sum_{k=-\infty}^{1} 2^{k u_{1} \frac{1}{p_{1}}} .
\end{aligned}
$$

That is to say, $f \in\left(\dot{K}_{u_{1}}^{\alpha_{1}, p_{1}}, l^{q_{1}}\left(\langle z\rangle^{s_{1}}\right)\right)$ if and only if $\alpha_{1} p_{1}<n .\|\mathcal{F} f\|_{\dot{K}_{u_{2}}^{\alpha_{2}, p_{2}} B_{q_{2}}^{s_{2}}}<\infty$ forces $p_{2}(n-$ $\left.a_{1}\right)>n$. From this it follows that $\frac{n}{p_{1}} \leq n-\frac{n}{p_{2}}$, which is equivalent to $p_{1} \geq p_{2}^{\prime}$.

Proposition 3.7 Let $1 \leq p_{1} \leq \infty, 0<p_{2}, q_{1}, q_{2}, u_{1}, u_{2} \leq \infty, \alpha_{1} \geq 0$, and $\alpha_{2}, s_{1}, s_{2} \in \mathbf{R}$. If

$$
\|\mathcal{F} f\|_{\dot{K}_{u_{2}}^{\alpha_{2}, p_{2}} B_{q_{2}}^{s_{2}}} \leq c\|f\|_{\left(\dot{K}_{u_{1}}^{\alpha_{1}, p_{1}, q_{1}}\left(\langle z)^{s_{1}}\right)\right)},
$$

then $s_{2} \leq \alpha_{1}+\alpha_{2}+s_{1}+\frac{n}{q_{1}}-\frac{n}{p_{2}^{\prime}}$.

Proof of Proposition 3.7 We estimate $\left\|\phi_{j}\right\|_{\left(\dot{K}_{u_{1}}^{\alpha_{1}, p_{1}, q_{1}\left(q_{1}(z)^{\left.\left.s_{1}\right)\right)}\right.}\right.}$ and $\left\|\mathcal{F} \phi_{j}\right\|_{\dot{K}_{u_{2}}^{\alpha_{2}, p_{2}} B_{q_{2}}^{s_{2}}}$. We have

$$
\begin{aligned}
&\left\|\phi_{j}\right\|_{\left(\dot{K}_{u_{1}, l q_{1}}^{\alpha_{1}, p_{1}}, q_{1}\left(\langle z\rangle^{s_{1}}\right)\right)}\left(\sum_{z}\langle z\rangle^{s_{1} q_{1}}\left\|\phi_{j} \mathcal{X}_{Q_{z}}\right\|_{\dot{K}_{u_{1}}^{\alpha_{1}, p_{1}}}^{q_{1}}\right)^{\frac{1}{q_{1}}} \\
&=\left(\sum_{k \leq j+1} 2^{k s_{1} q_{1}} \sum_{|z| \sim 2^{k}}\left\|\phi_{j} \mathcal{X}_{Q_{z}}\right\|_{\dot{K}_{u_{1}}^{\alpha_{1}, p_{1}}}^{q_{1}}\right)^{\frac{1}{q_{1}}} \\
& \backsim\left(\sum_{k \leq j+1} 2^{k s_{1} q_{1}} \sum_{|z| \sim 2^{k}} 2^{k \alpha_{1} q_{1}}\right)^{\frac{1}{q_{1}}} \\
& \backsim\left(\sum_{k \leq j+1} 2^{k s_{1} q_{1}} 2^{k n} 2^{k \alpha_{1} q_{1}}\right)^{\frac{1}{q_{1}}} \backsim 2^{j\left(s_{1}+\alpha_{1}+\frac{n}{q_{1}}\right)}, \\
&\left\|\mathcal{F} \phi_{j}\right\|_{\dot{K}_{u_{2}}^{\alpha_{2}, p_{2}} B_{q_{2}}^{s_{2}}} \backsim\left\|2^{j s_{2}} \mathcal{F} \phi_{j}\right\|_{\dot{K}_{u_{2}}^{\alpha_{2}, p_{2}}} \\
& \sim 2^{j s_{2}} 2^{j n}\left\|\mathcal{F} \phi_{1}\left(2^{j-1} x\right)\right\|_{\dot{K}_{u_{2}}^{\alpha_{2}, p_{2}}} \\
& \sim 2^{j s_{2}} 2^{j n} 2^{-j\left(\alpha_{2}+\frac{n}{p_{2}}\right)}\left\|\mathcal{F} \phi_{1}(x)\right\|_{\dot{K}_{u_{2}}^{\alpha_{2}, p_{2}}} \\
& \sim 2^{j\left(s_{2}-\alpha_{2}+\frac{n}{p_{2}^{\prime}}\right)} .
\end{aligned}
$$

Here we use $\|f(\lambda x)\|_{\mathcal{K}_{q}^{\alpha, p}}=\lambda^{-\left(\alpha+\frac{n}{p}\right)}\|f\|_{\dot{K}_{q}^{\alpha, p}}$ (see [15]); through $2^{j\left(s_{2}-\alpha_{2}+\frac{n}{p_{2}^{\prime}}\right)} \leq 2^{j\left(s_{1}+\alpha_{1}+\frac{n}{q_{1}}\right)}$ we have $s_{2} \leq \alpha_{1}+\alpha_{2}+s_{1}+\frac{n}{q_{1}}-\frac{n}{p_{2}^{\prime}}$. This completes the proof of Proposition 3.7. 
Competing interests

The authors declare that they have no competing interests.

\section{Authors' contributions}

All authors contributed equally to the writing of this paper. All authors read and approved the final manuscript.

\section{Acknowledgements}

Project (Grant Nos. 11261055, 11161044) was supported by NSFC, and SF of Xinjiang (Grant Nos. 2011211A005, BS120104).

Received: 20 January 2014 Accepted: 11 July 2014 Published: 19 Aug 2014

\section{References}

1. Stein, EM: Interpolation of linear operators. Trans. Am. Math. Soc. 83, 482-492 (1956)

2. Herz, C: Lipschitz spaces and Bernstein's theorem on absolutely convergent Fourier series. J. Math. Mech. 18, 283-324 (1968)

3. Kurtz, DS: Littlewood-Paley and multiplier theorems on weighted $L_{p}$ spaces. Trans. Am. Math. Soc. 259, 235-254 (1980)

4. Taibleson, $\mathrm{MH}$ : On the theory of Lipschitz spaces of distributions on Euclidean $n$-space. I. Principal properties. J. Math. Mech. 13, 407-479 (1964)

5. Sawano, Y, Yoneda, T: On the Young theorem for amalgams and Besov spaces. Int. J. Pure Appl. Math. 36(2), 197-205 (2007)

6. Lu, S, Yang, D: The decomposition of the weighted Herz spaces and its applications. Sci. China Ser. A 38(2), 147-158 (1995)

7. Triebel, H: Theory of Function Spaces. Monographs in Math., vol. 78. Birkhäuser, Basel (1983)

8. Xu, J, Yang, D: Herz-type Triebel-Lizorkin spaces I. Acta Math. Sin. 21(3), 643-654 (2005)

9. Triebel, H: Theory of Function Spaces II. Monographs in Math. Birkhäuser, Basel (1992)

10. Hedberg, L, Netrusov, Y: An axiomatic approach to function spaces, spectral synthesis and Luzin approximation. Mem. Am. Math. Soc. 188, 882 (2007)

11. Liang, Y, Yang, D, Yuan, W, Sawano, Y, Ullrich, T: A new framework for generalized Besov-type and Triebel-Lizorkin-type spaces. Diss. Math. 489, 1-114 (2013)

12. Ho, K: Littlewood-Paley spaces. Math. Scand. 108, 77-102 (2011)

13. Holland, F: Harmonic analysis on amalgams. J. Lond. Math. Soc. (2) 10, 295-305 (1975)

14. Fournier, JJF: On the Hausdorff-Young theorem for amalgams. Monatshefte Math. 95, 117-135 (1983)

15. Miyachi, A: Remarks on Herz-type Hardy spaces. Acta Math. Sin. Engl. Ser. 17, 339-360 (2001)

10.1186/1029-242X-2014-296

Cite this article as: Zhou and Cao: The boundedness of Fourier transform on the Herz type amalgams and Besov spaces. Journal of Inequalities and Applications 2014, 2014:296

\section{Submit your manuscript to a SpringerOpen ${ }^{\circ}$ journal and benefit from:}

- Convenient online submission

- Rigorous peer review

- Immediate publication on acceptance

Open access: articles freely available online

- High visibility within the field

- Retaining the copyright to your article 